\title{
Maternal Satisfaction and Associated Factors Among Pregnant Women Attended at Antenatal Care Service in Bedessa Health Center, Wolaita Zone, Ethiopia, 2018
}

\author{
Kelemu Abebe Gelaw*, Natnael Atnafu Gebeyehu \\ Department of Midwifery, College of Health Science and Medicine, Wolaita Sodo University, Wolaita Sodo, Ethiopia \\ Email address: \\ kelemuabebe2014@gmail.com (K. A. Gelaw), Natnaelatnafu89@gmail.com (N. A. Gebeyehu) \\ ${ }^{*}$ Corresponding author \\ To cite this article: \\ Kelemu Abebe Gelaw, Natnael Atnafu Gebeyehu. Maternal Satisfaction and Associated Factors Among Pregnant Women Attended at \\ Antenatal Care Service in Bedessa Health Center, Wolaita Zone, Ethiopia, 2018. Science Research. Vol. 8, No. 2, 2020, pp. 39-44. \\ doi: $10.11648 /$ j.sr.20200802.12
}

Received: September 6, 2019; Accepted: April 7, 2020; Published: April 28, 2020

\begin{abstract}
Background: Client satisfaction is indispensable for further advancement of the quality of focused antenatal care service and women's satisfaction has been directly linked to the quality of services given. This is essential to meet specific client needs and to provide uniform health care services for pregnant women. Objective:-To assess maternal satisfaction and associated factors among pregnant women attending Antenatal care service in Bedessa Health center, Wolaita Zone, Ethiopia, 2018. Methods: Facility-based cross-sectional study using quantitative methods was conducted among 387 pregnant women's. Subjects were selected using systematic random sampling technique. Data were coded, cleaned and entered by using Epi info version 7.2 and analyzed using SPSS version 20, and Bivariate and multiple logistic regression were carried out to identify factors associated with client satisfaction on antenatal care services and strength of association were declared at significance level of 0.05 . Result: More than half of the respondents (64.1\%) were satisfied with the service that they received. Those pregnant mothers who were merchants were three times more likely satisfied than housewife's (AOR $=2.649,95 \% \mathrm{CI}$ : $1.008,6.96)$. Mothers who had first visit ANC were almost three times more likely satisfied than those who had a fourth visit ( $\mathrm{AOR}=2.85,95 \% \mathrm{CI}$ : $1.383,5.90)$ and mothers who had second visits were two times more likely satisfied than those who had four visits ( $\mathrm{AOR}=2.14,95 \% \mathrm{CI}: 1.09,4.21$ ). Conclusions and Recommendation: The level of satisfaction was lower compared to other studies. Client feedback should be recognized as a legitimate method of evaluating health services in the health center as a whole.
\end{abstract}

Keywords: Antenatal Care, Maternal Satisfaction, Pregnant Women

\section{Introduction}

Satisfaction is the degree to which the client's desired expectations, goals and or preferences are met by the healthcare provider and/or service [1]. Women's satisfaction is an integral part of the quality assurance process with the potential to improve antenatal health services and has been linked to the quality of services given and the extent to which specific needs are me [2]. Antenatal care (ANC) refers to the comprehensive health supervision that is given to a pregnant woman from the time that conception is confirmed until the beginning of labor. It is important in preventing, monitoring, early detecting, and treating health problems for pregnant women and her family $[2,3]$.
According to United nation interagency estimates, maternal mortality refers to deaths due to complications from pregnancy or childbirth. From 1990 to 2015, the global maternal mortality ratio declined by 44 percent from 385 deaths to 216 deaths per 100,000 live births this translates into the average annual rate of reduction of 2.3 percent. While impressive, this is less than half the 5.5 percent annual rate needed to achieve the three-quarters reduction in maternal mortality [4].

Worldwide, approximately 830 women died every single day due to complications during pregnancy or childbirth among this causes the five major direct causes are hemorrhage 
(35\%), hypertensive disorders (18\%), unsafe abortions (9\%), sepsis $(8 \%)$, and prolonged or obstructed labor. Most of these conditions can be prevented with proper medical monitoring, information, and services [5]. Moreover, the majority of the countries with the highest maternal mortality are in sub-Saharan Africa, including Ethiopia. In sub-Saharan Africa and South Asia region, fewer women received at least four antenatal visits (49\% and $42 \%$ respectively) $[6,7]$.

According to Ethiopian Demographic Health Survey (EDHS), 2016 the level of maternal mortality and morbidity are among the highest in the world and the current estimate of Maternal Mortality Rate (MMR) is 412 per 100,000 live birth MMR for the 7-year period preceding the surveys and $80 \%$ of maternal deaths result from direct complications of pregnancy and childbirth [7, 8, 9]. In addition, only $34 \%$ and $62 \%$ of women who gave birth in the five years preceding the survey received antenatal care from a skilled provider respectively. Furthermore the study reviled that one woman in every five $(19 \%)$ made four or more antenatal care visits during the course of her pregnancy $[8,9]$ The existing evidence from developing countries including Ethiopia indicates that few women seek antenatal care at early stage of their pregnancy [6, 8] and only 19\% made their first ANC visit before the fourth month of pregnancy.

The study was done in Jimma town, Jimma zone, South West Ethiopia showed that $60.4 \%$ pregnant women's were satisfied with FANC service that they received. From the total respondents, most of the respondents $(80.7 \%)$ were satisfied with interpersonal aspects, and $62.2 \%$ were satisfied with organization of health care aspect. However, $49.9 \%$ of the respondents were not satisfied with technical quality aspect and $67.1 \%$ were not satisfied with physical environment aspect [4]. Most maternal deaths are preventable as the necessary medical interventions are well known, therefore crucially important to increase women s access to quality care before, during and after childbirth. The Ending Preventable Maternal Mortality Strategy (EPMMS) suggests adaptive highly effective interventions to improve women health, before, during, and after pregnancy $[6,7,8]$.

The global strategies (2016-2030) is roadmap to achieve right to the highest attainable standard of health for all women to transform the future and ensure every mother not only survives but thrives The Ending Preventable Maternal Mortality (EPMM) target's and strategies are focus on eliminating significant inequities that led to disparities in access, quality and outcomes of care within and between countries. Concrete political commitments and financial investments by country governments and development partners are necessary to meet the targets and carry out the strategies for ending preventable maternal mortality (EPMM) [10].

Despite the fact that maternal ANC service satisfaction level is important for further improvement of maternal and child health, maternal ANC service satisfaction was not studied especially in study area. Therefore the study was aimed at assessing maternal ANC service satisfaction and associated factors and the finding of this study might be key to the improvement in the quality of care, scaled up the understanding of the pattern of demand and uptake of maternity services among pregnant women, health care planners might use the information generated from the study to improve service delivery dimension and quality among pregnant women attending antenatal follow-up visit. The findings of this study also might also be served as a reference for giving intervention to the health care providers and others for conducting further researches. In addition to this, it had special importance for health care providers to serve as baseline information for filling gaps of the actual practices on the quality of antenatal services.

\section{Method and Materials}

\subsection{Study Area and Period}

The study was conducted at Bedessa town health centers in 2018. Bedessa Town is found in Damot Waide Woreda and is capital of Damot Waide woreda which is established in 1957E.C. The town is $374 \mathrm{~km}$ southwest from the capital city of Ethiopia which is Addis Ababa and it is $102 \mathrm{~km}$ far from Hawassa, the capital city of SNNRP and $26 \mathrm{~km}$ north-east from Wolaita Sodo, the capital of Wolaita zone. It has one primary school and one secondary and preparatory school, two health posts and One Health Center [11]. The study was conducted from April 1 to July 30, 2018.

\subsection{Study Design}

A facility-based cross-sectional study design with quantitative methods of data collection was employed.

\subsection{Source Population}

All pregnant women attending antenatal care service in health centers at Bedessa town in 2018.

\subsection{Study Population}

All pregnant women attending antenatal care service in the selected health center at Bedessa town.

\subsection{Study Unit}

Individual pregnant women who came for ANC follow up visit.

\section{Inclusive criteria}

All pregnant women who came for ANC follow up visit during the data collection period.

Exclusive criteria:

Mothers who are seriously ill and unable to respond were excluded.

\section{Sample size determination:}

The sample size was calculated by using the single population proportion formula by considering $60.4 \%$ proportion of respondents satisfaction of study done in, Jimma zone, South West Ethiopia (4). With a marginal error of 5\% between the sample and the population at $95 \%$ confidence level so, the sample size was determined as follows:- 


$$
\mathrm{n}=\frac{(Z \propto / 2)^{2} \times p(1-p)}{d^{2}}
$$

Whereas;

$\mathrm{n}=\mathrm{n}$ is the minimum sample size required

$\mathrm{Z}=$ is the standard normal value at (1-alpha)

Alpha is 5\% with CI 95\%

$\mathrm{P}=$ Proportion of women taken from previous study

$\mathrm{d}=\mathrm{d}$ is the margin of sampling error tolerated (5\%)

$$
\mathrm{n}=\frac{(1.96)^{2} \times 0.604(1-0.604)}{(0.05)^{2}}=368
$$

By adding 5\% non-response rate, the total sample size was 387.

\section{Sampling technique:}

Systematic sampling technique was employed to select study subjects in the study area. The total sample size was proportionally allocated to the selected health center and health posts and participants were taken every $\mathrm{k}=\mathrm{N} / \mathrm{n}=2$ interval. The first client to be included in the study was selected by lottery method and then every 2 participants were taken.

Dependent variables

Client satisfaction

Independent variables:

I. Socio-demographic variables (age, religion, residence, ethnicity, marital status, occupation, educational level) II. Obstetric and gynecological related factors (parity, number of pregnancy, type of pregnancy, history of abortion, history of stillbirth, number of antenatal visits)

II. Health center and provider-related factors: (explaining the result of the examination, advice on danger sign during pregnancy, availability of laboratory test

Data collection instrument and techniques:

The data were collected using pre-tested structured questionnaires and the questionnaire contains items which were related to socio-demographic characteristics of the respondents, obstetric and gynecological related factors; current health center and provider-related factors and to measure maternal satisfaction about antenatal care service in health centers. A 5-point Likert scale ranging from dissatisfied to fully satisfied ( 1 to 5 points) items were used to assess the outcome variable.

Data collection procedures:

Data were collected from April 1 to July 30, 2018 using six data collectors ( 2 diploma midwives and 2 diploma nurses)) for data collection and $2 \mathrm{BSc}$ midwives as supervisors. One day data collection training was given for data collectors and supervisors concerning the aim of the study, contents of the questionnaire, issues of confidentiality and privacy and questionnaires were filled by direct face to face interview.

Data processing and analysis:

Once the collected data were checked for completeness and consistency, it was coded, cleaned and entered into Epi-info version 7.2 for data template and then exported to SPSS version 20 for analysis. Descriptive statistics were computed to see the frequency distribution and presented in terms of frequency, mean, median and standard deviation. To compute overall satisfaction rate, mothers who were scored greater or equal to $75 \%$ of the items were categorized under "satisfied" and those who were scored less than $75 \%$ of the items were categorized as "dissatisfied" $[3,4]$. Both binary and multivariate logistic regression analyses were carried out to appreciate the association between the independent and dependent variables. Variables with p-value $<0.02$ in Bivariate logistic regression model were entered to multivariate analysis model to control the presence of potential confounding factors and its p-value $<0.05$ were taken as a cut of point to declare statically significant to this study. Finally, the findings were presented in texts, tables, and graphs.

Data quality control measures:

A pre-test was conducted on $5 \%$ of the sample (20 pregnant women's) from the health center other than the study area. The questionnaire and informed consent were prepared in English and translated into local Wolaitigna language and re-translated back to English to keep its consistency. Close supervision was undertaken during data collection and every questionnaire was cross-checked daily after data collection.

Operational definition:

Client satisfaction: The extent of an individual's experience compared with his or her expectations.

Satisfaction: It was measured based on five Likert scales (strongly disagree, disagree, neutral, agree and strongly agree) and this was finally categorized as satisfied and dissatisfied. The responses strongly agree and agree are classified as satisfied and strongly disagree, disagree and neutral were categorized as not satisfied.

Overall satisfaction level: respondents who score $\geq 75 \%$ of the $11^{\text {th }}$ satisfaction indicator items were categorized under "satisfied" and those who score were less than $75 \%$ of the items were categorized as "not satisfied" $[3,4]$.

\section{Results}

Socio-demographic characteristics of the respondents:

In this study, three hundred fortify (340) mothers were interviewed/participated in giving a response rate of $88 \%$. Near three fourth $(77.3 \%)$ of the respondents were in the age group of 20-34 years with a median age of 25.5 years, ranging a minimum 15 years to maximum 45 years. From participants, three-hundred-sixty-five $(94.3 \%)$ of them were married. Majority $250(64.6 \%)$ of the study population was protestant and $267(69 \%)$ were coming from urban. Two hundred seventy-seven (277 (71.6\%)) were housewives and 225 (58.1) of them were educated (table 1).

Table 1. Socio-demographic characteristics of pregnant women attending ANC at Health Centers, Bedessa town, Wolaita Zone, Ethiopia, 2018.

\begin{tabular}{lllll}
\hline S.no & Variables & Category & Frequency (n) & Percent (\%) \\
\hline & & $<20$ & 47 & 12.1 \\
1. & Age in years & $20-34$ & 77.3 & 299 \\
& & $>35$ & 41 & 10.6 \\
2. & Marital status & Married & 94.3 & 365 \\
\hline
\end{tabular}




\begin{tabular}{|c|c|c|c|c|}
\hline S.no & Variables & Category & Frequency (n) & Percent (\%) \\
\hline \multirow{4}{*}{3.} & \multirow{4}{*}{ Educational status } & Single & 22 & 5.7 \\
\hline & & Can't read and write & 162 & 41.9 \\
\hline & & Primary & 181 & 46.7 \\
\hline & & Secondary and above & 44 & 11.4 \\
\hline \multirow{2}{*}{4.} & \multirow{2}{*}{ Residence } & Urban & 267 & 69 \\
\hline & & Rural & 120 & 31 \\
\hline \multirow[t]{4}{*}{5.} & \multirow[t]{4}{*}{ Religion } & Protestant & 250 & 64.6 \\
\hline & & Orthodox & 95 & 24.5 \\
\hline & & Muslim & 20 & 5.7 \\
\hline & & Catholic & 22 & 5.7 \\
\hline \multirow[t]{3}{*}{6.} & \multirow[t]{3}{*}{ Ethnicity } & Wolaita & 329 & 85 \\
\hline & & Gamogofa & 46 & 11.9 \\
\hline & & Others* & 12 & 3.1 \\
\hline \multirow[t]{4}{*}{7.} & \multirow[t]{4}{*}{ Occupation } & Housewife & 277 & 71.6 \\
\hline & & Employee & 55 & 14.2 \\
\hline & & Merchant & 36 & 9.3 \\
\hline & & Student & 19 & 4.9 \\
\hline
\end{tabular}

Obstetric and gynecologic characteristic of the respondents:

From the total studied participants, $323(83,5 \%)$ of them have given birth for one to four children followed by never given birth and who gave birth for five or more children accounting $51(13.2 \%)$ and $13(3.3 \%)$ respectively. From those who given birth $336(86.8 \%), 10$ (2.6\%) of them end up with stillbirths. Thirty-five (9\%) of the women had a previous history of abortions. The majority of the respondents' 333 $(86 \%)$ agreed that their current pregnancy is planned and wanted (Table 2).

Table 2. Obstetric and Gynecologic characteristics of the respondents attended ANC at Health centers, Bedessa town, Wolaita Zone, Ethiopia, 2018.

\begin{tabular}{|c|c|c|c|c|}
\hline S no & Variables & Category & Frequency (n) & Percentage (\%) \\
\hline \multirow{5}{*}{1.} & \multirow{5}{*}{ Number pregnancy } & 1 & 55 & 14.2 \\
\hline & & 2 & 113 & 29.2 \\
\hline & & 3 & 106 & 27.4 \\
\hline & & 4 & 58 & 15 \\
\hline & & $>5$ & 55 & 14.2 \\
\hline \multirow{4}{*}{2.} & \multirow{4}{*}{ parity } & Nulliparous & 51 & 13.2 \\
\hline & & Multipara & 323 & 83.5 \\
\hline & & Grand multipara & 13 & 3.3 \\
\hline & & Total & 387 & 100 \\
\hline \multirow{2}{*}{3.} & \multirow{2}{*}{ Current Visit } & First & 112 & 28.9 \\
\hline & & Follow up visit & 275 & 71.1 \\
\hline \multirow{2}{*}{4.} & \multirow{2}{*}{ Type of pregnancy } & Unplanned & 54 & 14 \\
\hline & & planned & 333 & 86 \\
\hline \multirow{2}{*}{5.} & \multirow{2}{*}{ History of abortion } & No & 352 & 91 \\
\hline & & $\mathrm{Had}$ & 35 & 9 \\
\hline 6. & History of stillbirth & No & 377 & 97.4 \\
\hline
\end{tabular}

Pregnant women's satisfaction regarding focused ANC:

Client satisfaction was assessed by 11 item satisfaction questions. According to this study, 248 (64.1\%) of the mothers participated in the study were satisfied. Eighty-seven percent $(87.9 \%)$ and $(85.3 \%)$ of women were highly satisfied with provider performed Laboratory investigation and Care provision respectively. However, $(82.2 \%)$ and $(31 \%)$ of the respondents reported absence of providers routine physical examinations and explanation of the results of examination the main reasons for dissatisfaction with the overall quality of care received in the health centers respectively. Also, $32.8 \%$ of them were dissatisfied with the explanations of danger sign arise during pregnancy (Table 3 ).

Table 3. Pregnant women's overall satisfaction regarding focused ANC in Health centers in Bedessa town, Wolaita Zone, Southern, Ethiopia, 2018.

\begin{tabular}{llll}
\hline S. No & Components & Satisfied & Dissatisfied \\
\hline 1. & Greets and calls the client by her name and introduce her /himself/ & $303(78.3)$ & $84(21.7)$ \\
2. & Reviews clinic record before starting the session and check about previous pregnancy, number, and outcome & $302(78)$ & $85(22)$ \\
3. & Provider treat you respectfully & $326(84.2)$ & $61(15.8)$ \\
4. & Provider perform general examination & $69(17.8)$ & $318(82.2)$ \\
5. & The provider performs Laboratory investigations & $340(87.9)$ & $47(12.1)$ \\
6. & Health care provision like- iron/foliate supplementation, Tetanus toxoid immunization. & $330(85.3)$ & $57(14.7)$ \\
7. & Care that you want and need & $321(82.9)$ & $(17.1)$ \\
8. & Return to your home without having a check up & $322(83.2)$ & $65(16.8)$ \\
9. & Result of examination & $167(69)$ & $120(31)$ \\
10. & All the advice you have got & $296(76.5)$ & $91(23.5)$ \\
11. & Explanations of danger sign arise during pregnancy & $260(67.2)$ & $127(32.8)$ \\
\hline
\end{tabular}


Factors associated with satisfaction of pregnant women's regarding FANC:

Both bivariate and multivariate logistic regression analysis were made to identify predictors of satisfaction. The bivariate analysis result revealed that socio-demographic variables such as occupation, religion, obstetric profile variables such as a number of ANC visit were significantly associated with satisfaction with focused antenatal care services. These variables were entered into a multivariate logistic regression to control confounding. The variables with $p$-value less than
0.05 in multivariate analysis were taken as significant predictors of satisfaction and the rest were refuted. Those pregnant mothers who were merchants were three times more likely satisfied than housewife's $(\mathrm{AOR}=2.649,95 \% \mathrm{CI}$ : 1.008 , 6.96). Mothers who had first visit ANC almost three times more satisfied than those who had fourth visit $(\mathrm{AOR}=2.85$, 95\%CI: $1.383,5.90)$ and mothers who had second visits two times more satisfied than those who had four visits $(\mathrm{AOR}=2.14,95 \% \mathrm{CI}: 1.09,4.21)$ (Table 4).

Table 4. Associations between socio-demographic characteristics and obstetric-related factors with the satisfaction of mothers on FANC, Bedessa town, Wolita, Ethiopia, 2018.

\begin{tabular}{|c|c|c|c|c|c|c|}
\hline \multirow{2}{*}{ Variables } & \multirow{2}{*}{ Category } & \multicolumn{2}{|c|}{ Overall satisfaction } & \multirow{2}{*}{ COR $(95 \% \mathrm{CI})$} & \multirow{2}{*}{ AOR $(95 \% C I)$} & \multirow{2}{*}{ P-value } \\
\hline & & Dissatisfied (\%) & Satisfied (\%) & & & \\
\hline \multirow{4}{*}{ Occupation } & Employee & $11(7.9)$ & $8(3.2)$ & $1.913(0.665,5.503)^{*}$ & $1.659(0.565,4.870)$ & 0.0229 \\
\hline & Student & $18(12.9)$ & $18(7.3)$ & $1.375(0.448,4.218)$ & $1.268(0.405,3.969)$ & 0.578 \\
\hline & Merchant & $87(62.6)$ & $190(76.6)$ & $3.003(1.167,7.729) *$ & $2.649(1.008,6.956)^{* *}$ & 0.023 \\
\hline & Housewife & $23(16.5)$ & $32(12.9)$ & 1.00 & 1.00 & 1.00 \\
\hline \multirow{3}{*}{$\begin{array}{l}\text { Number of } \\
\text { ANC visit }\end{array}$} & First visit & $29(20.9)$ & $83(33.5)$ & $2.862(1.399,5.857)^{*}$ & $2.857(1.383,5.900)^{* *}$ & 0.005 \\
\hline & Second visit & $56(40.3)$ & $111(44.8)$ & $1.982(1.023,3.840)$ & $2.146(1.094,4.210)^{* *}$ & 0.026 \\
\hline & Fourth visit & $23(16.5)$ & $23(9.3)$ & 1.00 & 1.00 & 1.00 \\
\hline
\end{tabular}

N.B * P-value $<0.025, * *$ P-value $<0.05$

\section{Discussion}

In this study, the overall satisfaction of pregnant mothers on focused antenatal care service in the study population was $64.1 \%$. This is fairly similar to the study conducted in Jimma (60.4\%) [4]. However, the finding was lower than the study conducted in Addis Ababa [13], Ethiopia (89.2\%), and greater than the study done in Nepal (45.1\%) [12] and Bursa (33\%) [3]. The difference might be due to the subjective nature of the participants as a result of using unstandardized too or yes/no to measure the satisfaction, the socio-demographic variables of the study population, or it might be due to time variation of the study period.

The finding of this study indicated that $(87.9 \%)$ and $(85.3 \%)$ of pregnant women were satisfied with laboratory investigation and care provision of focused antenatal care services respectively. However, half $(82.2 \%)$ and $23.5 \%$ of pregnant women were dissatisfied with general physical examination and advice on danger sign occur during pregnancy respectively. The perceived cause of dissatisfaction forwarded by the majority does not explain the result of the examination $(31 \%)$. This finding is somewhat different from the study conducted in Jimma which showed that $80.7 \%$ and $62.2 \%$ of pregnant women were satisfied with interpersonal and organization of health care aspects of focused antenatal care services respectively [4]. This might be due to the assessment too used and study design difference used in the previous study.

In this study; those pregnant mother who was merchants were three times more likely satisfied than housewife's $(\mathrm{AOR}=2.649,95 \% \mathrm{CI}: 1.008,6.96)$. The result of this study is not consistent with other study conducted in Debre Marcos town [14]. The possible reason why merchant women's were better satisfied were as they have better monthly income than others, they can easily access transportation and may access health-related issues than others. It was also observed that pregnant women who had ANC first visit were almost three times more likely satisfied than those who had fourth visit $(\mathrm{AOR}=2.85,95 \% \mathrm{CI}: 1.383,5.90)$ and mothers who had second visits were two times more likely satisfied than those who had four visits $(\mathrm{AOR}=2.14,95 \% \mathrm{CI}: 1.09,4.21)$. This finding is similar to the study conducted in Jimma town [4]. The similarities might be due to the health care providers concern on detail counseling, assessment, and examination in the first and second visits and their similar level of knowledge and skills regarding of focused ANC services.

\section{Conclusion}

Although, more than half of the study population (64.1\%) was satisfied with the focused antenatal care service in the study area, when compared with the previous studies conducted in different areas, their level of satisfaction was low. This indicates that a decrease in the clients' satisfaction is followed by a decrease in the quality of care level. In addition, the participants were dissatisfied on the absence of provider's routine physical examinations and explanation of their results after examination. So, Continuous monitoring of clients' satisfaction with all aspects of care could aid in improvement of the quality of services.

\section{Abbreviations}

ANC: Antenatal Care

EDHS: Ethiopia Demographic Health Survey

EPMM: Ending Preventable Maternal Mortality

FANC: Focused Antenatal Care

SNNP: Southern Nation, Nationality and People of 
Ethiopia

WHO: World Health Organization

\section{Declarations}

Ethics approval and consent to participant

Ethical clearance was obtained from Wolaita Sodo University, College of Health Sciences and Medicine. Personal patient information was not recorded, after finishing the data collection the patients' document return to the card room, the information used for study purpose only.

\section{Consent for Publication}

Not applicable.

\section{Availability of Data and Materials}

The data that support the findings of this study are available but some restrictions may apply to the availability of these data as there are some sensitive issues. However, data are available from the corresponding authors upon reasonable request.

\section{Competing Interests}

The authors declare that they have no competing interests.

\section{Funding}

Wolaita Sodo University

\section{Authors' Contributions}

KA was involved in the conception, design, analysis, interpretation, report, manuscript writing, design, analysis, interpretation and report writing. NA was involved in the design, analysis, and interpretation of the data. All authors read and approved the final manuscript.

\section{Acknowledgements}

We would like to express our deepest heartfelt thanks to Wolaita Sodo University for allowing conducting this study. Our special thanks go to Bedessa Health Center hospital staffs for their support during the data collection process.

\section{References}

[1] Nemat Ismail Abdel Aziz Ismail, Rasha Mohamed Essa Pregnant Women's Satisfaction with the Quality of Antenatal Care At Maternal and Child Health Centers in El-Beheira Governorate. IOSR Journal of Nursing and Health Science. (Mar. - Apr. 2017), Volume 6, Issue 236-46.
[2] Marzhan A. Dauletyarova 1, Yuliya M. Semenova. Are Kazakhstani Women Satisfied with Antenatal Care? Implementing the WHO Tool to Assess the Quality of Antenatal Services. in 2017, 18: 2.

[3] Tesfaye T, Mekonnen H and Negesa L. Maternal Antenatal Care Service Satisfaction and Factors Associated with Rural Health Centers, Bursa District, Sidama Zone, Southern Ethiopia: A Cross-sectional Study. Journal of Women's Health Care 2017, 6: 2.

[4] Fantaye Chemir, Fessahaye Alemseged, and Desta Workneh. Satisfaction with focused antenatal care service and associated factors among pregnant women attending focused antenatal care at health centers in Jimma town, Jimma zone, South West Ethiopia; a facility-based cross-sectional study triangulated with a qualitative study. BMC. 2014, 7: 164.

[5] World Health Statistics. Monitoring Health for the Sustainable Development Goals (SDGs).2017.

[6] UNICEF Data: Monitoring the Situation of Children and Women.

[7] International. I. Central Statistical Agency EDHS, Addis Ababa 2016.

[8] Ethiopia Demographic and Health Survey 2016. Addis Ababa, Ethiopia, and Calverton, Maryland, USA: Central statistical agency and ICF Macro.

[9] Leontine Alkema, Doris Chou, Daniel Hogan, on behalf of the United Nations Maternal Mortality Estimation Inter-Agency Group collaborators and technical advisory group. Global, regional, and national levels and trends in maternal mortality between 1990 and 2015, with scenario-based projections to 2030: a systematic analysis by the UN Maternal Mortality Estimation Inter-Agency Group. World Health Organization, Department of Reproductive Health. November 12, 2015.

[10] The Maternal mortality Report falls between 1990 to 2015, United Nations, 2013; UNDP, UNFPA; UNICEF; UN Women; WHO.

[11] Mekonnen N, Berheto TM, Ololo S, et al. Quality of Antenatal Care Services in Demba Gofa Woreda, Gamo Gofa Zone, Rural Ethiopia. Health Sci J 2017, 11: 3

[12] Sadiksha Regmi, Hari Prasad Kaphle, Neena Gupta. Maternal satisfaction regarding perinatal care and influencing factors in tertiary hospitals of western, Nepal. 2017, 06: 8595.

[13] Tesfaye T, Maternal Antenatal Care Service Satisfaction and Factors Associated with, in Addis Ababa Ethiopia 2014: A Cross-sectional Study Tesfaye et al., J Women's Health Care. 2017, 6: 2.

[14] Kurabachew Bitew, Mekonnen Ayichiluhm, and Kedir Yimam. Maternal Satisfaction on Delivery Service and Its Associated Factors among Mothers Who Gave Birth in Public Health Facilities of Debre Markos Town, Northwest Ethiopia; volume 2015 (6). 